\title{
Evaluación para el mejoramiento de la formación docente en educación infantil: Reto de la profesionalización del servicio educativo
}

\author{
Carmen Aura Arias Castilla y María de Jesús Blanco Vega*
}

Resumen. Este artículo tiene como objetivo describir un tipo de evaluación de saberes de madres comunitarias en profesionalización para el desarrollo infantil, como medio de valoración de la atención a la primera infancia en Colombia. El alcance es descriptivo con enfoque mixto. La población está conformada por un grupo de madres comunitarias de Hogares infantiles comunitarios. Como técnica investigativa se utilizó la entrevista a grupo focal, mediante instrumento validado por expertos y orientado a la indagación de los ejes nucleares de la cualificación del talento humano desde lineamientos técnicos para atención educativa infantil. Los resultados están relacionados con los saberes de las madres comunitarias de Programa de Pedagogía Infantil en temáticas específicas de la educación infantil: concepción de infancia, conocimiento sobre política pública de infancia, gestión de proyectos pedagógicos, inter-sectorialidad en servicios integrales, didáctica y estrategias pedagógicas desarrolladas para la atención a la primera infancia en el desarrollo de la profesionalización.

Palabras clave: formación profesional, profesionalización, infancia, conocimientos, práctica docente.

AVALIAÇÃO PARA A MELHORIA DA FORMAÇÃO DE PROFESSORES EM EDUCAÇÃO INFANTIL: DESAFIO DA PROFISSIONALIZAÇÃO DO SERVIÇO EDUCATIVO

Resumo. Este artigo tem como objetivo descrever um tipo de avaliação do conhecimento das mães comunitárias na profissionalização para o desenvolvimento infantil, como forma de avaliar os cuidados na primeira infância na Colômbia. O escopo é descritivo com uma abordagem mista. A população é composta por um grupo de mães de lares de crianças da comunidade. A técnica de pesquisa utilizada foi uma entrevista de grupo focal, mediante um instrumento validado por especialistas e focado na investigação dos eixos nucleares da qualificação do talento humano a partir de orientações técnicas para a educação infantil. Os resultados estão relacionados com o conhecimento das mães da comunidade do Programa de Pedagogia Infantil em temáticas específicas de educação infantil: a concepção de infância, o conhecimento sobre políticas públicas para as crianças, a gestão de projetos pedagógicos, a intersetorialidade em ser-

\footnotetext{
* Corporación Universitaria Iberoamericana, Colombia.
} 
viços abrangentes, didática e estratégias pedagógicas desenvolvidas para o cuidado da primeira infância no desenvolvimento da profissionalização.

Palabras clave: formação profissional; profissionalização; infância; conhecimento; prática docente.

EVALUATION FOR IMPROVING TEACHER TRAINING IN CHILDHOOD EDUCATION: THE CHALLENGE OF PROFESSIONALIZING EDUCATIONAL SERVICE

Abstract. This article describes a type of evaluation of knowledge of community mothers engage in a professionalization process for the child development, this, as a way of assessing early childcare in Colombia. The article used and mixed methods approach and has a descriptive scope. The targeted population is the mothers working at community childcare. Focus group interviews were used as a primary research technique. This instrument, validated by experts, aimed to investigate the nuclear axes of human talent qualification from the technical guidelines for childhood educational attention. The results are related to the knowledge of community mothers that participated in the of the Children's Pedagogy Program knowledge. Particularly on specific aspects of early childhood education such as: conceptualization of childhood, knowledge about child hood public policy, management of pedagogical projects, intersectoriality in childhood services, and didactic and pedagogic strategic for early childcare in the process of professionalization.

Keywords: professional training; professionalization; childhood; knowledge; teaching practice.

\section{INTRODUCCIÓN}

Numerosos grupos de investigación y programas de formación en pedagogía infantil, aciertan en identificar la importancia de los esfuerzos por mejorar la calidad de formación de tutores y tutoras de trabajo pedagógico con la infancia, también el interés manifiesto de indagar en las formas cómo aprenden las madres comunitarias, en adelante MC, y mejorar la calidad de la atención de los niños y niñas de primera infancia en Colombia, mediante la cualificación del talento humano en la profesionalización de los agentes educativos.

Describir los saberes y prácticas de los agentes educativos encargados del desarrollo y educación de la infancia es un reto de evaluación de la formación que a largo y mediano plazo traerá sus frutos para la educación infantil y el desarrollo de Colombia, en diferentes ámbitos de la vida de la nación.

Guzmán y Guevara (2010) afirman que "tanto las prácticas como las ideas que tienen los tutores y las tutoras acerca del trabajo con los niños y las niñas más pequeños, tienen una estrecha relación con sus concepciones de 
infancia" (p. 862), por ejemplo, una tendencia según estas autoras, afirma que a pesar de las teorías nuevas que se enseñan a los maestros y maestras en su ejercicio, ellos y ellas tienden a repetir, en sus prácticas cotidianas, las mismas formas de enseñanza en que fueron formadas por sus profesores. Esta afirmación conlleva un primer reto, transformar los parámetros educativos de infancia con el propósito de incorporar a docentes del servicio educativo infantil en las nuevas tendencias y concepciones de la niñez, respetar y preservar los derechos.

Otras tendencias, afirman que los maestros y maestras no ponen en práctica sus saberes y conocimientos sobre lo que han aprendido sobre la infancia, sino que lo hacen según sus creencias, "[...] es decir, se establecen a partir de lo que cada persona vive, escucha y en muchas ocasiones de lo que a cada uno le suele pasar" (Jaramillo, 2009, pág. 3)

Al contrario, otra de las perspectivas resalta como un aspecto importante de la política de primera infancia, es la conservación y aprovechamiento de las prácticas culturales.

Las madres comunitarias, agentes encargadas de la educación infantil, cumplen un rol vital en la apropiación de estas prácticas significativas para la educación infantil. Las MC identifican, utilizan y transforman prácticas culturales de su entorno en el desarrollo de los niños, en espacios significativos, definidos por Puche, Orozco y Otálora, 2010, citado por Hernández Muñoz (2013), cuando señalan que éstos se caracterizan por ser: "Escenarios de aprendizaje estructurado, retador y generador de múltiples experiencias para los niños y niñas que participan en él.(pág.5).

La visión del perfil de la MC desde el Instituto Colombiano de Bienestar Familiar, (Soto Panesso y Casanova Martínez, 2009, pág. 46), se relaciona con el concepto de la madre comunitaria como un actor importante dentro de las comunidades, donde se encuentran ubicados los hogares comunitarios, debe ser la madre comunitaria una persona: Directamente responsable de la atención humanizada de los niños y las niñas, debe poseer actitud y aptitud para el trabajo con la infancia, mayor de edad, menor de 55 años, reconocido comportamiento social y moral, como mínimo, que posea vivienda adecuada o que tenga disposición para atender a los niños y niñas en espacios comunitarios; que acepte su vinculación al programa como un trabajo solidario y voluntario, dispuesta a capacitarse para brindar una mejor atención a los infantes beneficiarios, que goce de buena salud, disponga del tiempo necesario para dedicarse a la atención de los menores y contar con la acogida dentro de la comunidad. 
Las MC en profesionalización, son agentes educadoras en formación, cuyas edades oscilan en edades entre los 20 y 60 años de edad, poseen gran interés de formación, dado que han obtenido títulos de formación técnico y tecnológico y tienen el propósito de seguir cualificándose a nivel profesional para brindarle una mejor atención a la infancia. (Blanco Vega y Arias Castilla, 2015).

Las denominaciones de las hoy llamadas madres comunitarias han variado de los nombres asociados a "personas encargadas de cuidar y cocinar", hasta el nombre actual y técnico de "agentes educativos", título al cual son asociadas a educar con soporte pedagógico. Las MC tienen gran incidencia en la vida de los niños que a diario reciben en sus hogares, es por eso que su rol va más allá de alimentar y protegerlos, también dedican su tiempo a educarlos y asegurar elementos de la salud (pesarlos y medirlos) relacionados con su desarrollo social, (ficha de valoración cualitativa del desarrollo psicológico) y formativo desde la realización de actividades pedagógicas, teniendo presente que desde 1990 se encuentran los orígenes del Proyecto Pedagógico Educativo comunitario - PPEC - del Instituto Colombiano de Bienestar Familiar ICBF.

\section{MARCO TEÓRICO}

Entender históricamente el rol de las maestras jardineras y más recientemente las agentes educadoras, nominadas como "madres comunitarias" así como su evolución en el conocimiento de sí mismas y del rol que tienen en la educación de la niñez y el respeto como sujetos de derechos, atiende a una forma innovadora de evaluar su formación.

El Programa de Proyecto Pedagógico Educativo Comunitario, en adelante PPEC, que se ha entendido con la educación de la primera infancia, tuvo su origen en los Hogares Comunitarios de Bienestar - HCB - y se configuró según Osorio Franco, Cortés Urrego y Rodas Mejía (2016), a partir de una "iniciativa de mujeres solidarias que de manera voluntaria se dieron a la tarea de cuidar los hijos de sus vecinos, mientras estos salían a trabajar, se configuró el programa Hogares Comunitarios de Bienestar HCB" (López Lara, 2014, pág. 24).

La formación de las MC, se ha realizado históricamente hacia su formación desde el inicio de los HCB, hasta el establecimiento de los PPEC, con el objetivo de formar verdaderos agentes educativos para mejorar la calidad educativa de la Pedagogía Infantil en Colombia; la formación a nivel técnico: Atención Integral a la primera infancia AIPI, con el objetivo 
siguiente: contribuir a la cualificación del personal vinculado a la atención a la primera infancia de modo que aportara al desarrollo integral de los niños y las niñas, y en consecuencia el avance económico, social y tecnológico del país [...] con el fin de elevar su calidad técnica pedagógica y cooperar en el proceso de protección de derechos de niñas, niños y sus familias, amparados bajo el programa del ICBF, citado por Osorio Franco, Cortés Urrego y Rodas Mejía (2016).

La formación a nivel profesional o profesionalización, se concibe como una experiencia transformadora, algunos estudios afirman que la importancia que le dan algunas MC que cursan la profesionalización, se centra, para algunas en la valoración de contenidos que le aportan y para otras, en la incidencia sobre su formación personal y profesional (Osorio Franco, Cortés Urrego y Rodas Mejía, 2016).

La profesionalización desde las políticas públicas estatales a nivel nacional e internacional, propende por mejorar la calidad de la educación y abordar una enseñanza eficaz, pensada desde el desarrollo cognitivo y socioafectivo y teniendo en cuenta los conocimientos previos del estudiante. Los estudios realizados han determinado que los procesos desarrollados en el aula son un elemento esencial en el desempeño académico de los estudiantes. Por lo tanto, las practicas pedagógicas, los comportamientos y las actitudes del docente o agente educativo inciden en la formación de la infancia y favorecen el desarrollo integral, en una perspectiva de equidad y calidad de vida (Martinez Garrido y Murillo, 2016).

Las transformaciones que se dan en la formación de las MC, en un pasado reciente, han sido desde la experiencia del estudio de Borjas, Jaramillo y Osorio (2009), en la investigación "Nichos pedagógicos" en varios frentes: alrededor del discurso pedagógico (vocabulario pedagógico, incorporación de lenguaje técnico referido a las prácticas pedagógicas); apropiación de saberes relacionados con contenidos del PPEC; en los componentes de la escala cualitativa de valoración: relaciones, procesos, momentos pedagógicos e indicadores; en relación al proceso de planeación de actividades: diseño y evaluación de estrategias; distinción e identificación de las características principales de las etapas de desarrollo infantil entre 3 y 5 años. Alrededor de las prácticas educativas, la transformación se hizo a propósito de la contextualización, las características del entorno, la planeación de momentos pedagógicos, sobre las actitudes y relaciones sociales, los avances fueron el mejoramiento en las relaciones sociales para permitir acercamiento y conocimientos entre las MC. En este estudio se recomendó: Propiciar procesos de planeación centrada en cada uno de los momentos pedagógicos y aumento del tiempo para profundizar en el proceso del Modelo Pedagógico. 
En términos de las prácticas pedagógicas la metodología es un elemento básico conformado por la didáctica, los contenidos, el diseño de actividades, los objetivos y la planificación como herramienta para enseñar. En la formación inicial docentes y agentes educativos buscan contemplar el contexto social y cultural de la infancia, a través del diseño y planeación de acciones y actividades pertinentes a sus edades. De ahí, la importancia de concretar las intencionalidades educativas, así como la selección de contenidos, secuencias y evaluación, en torno a un proceso de formación integral infantil (Hernández Cervantes y Blanch, 2014).

En la infancia, la enseñanza se enmarca en procesos de participación entre las diferentes instancias y actores como docentes, agentes educativos y familia, éstos son relevantes en el aporte al enfoque sociocultural reflejado en actividades desarrolladas en el aula. El trabajo colaborativo permite tomar decisiones, definir objetivos y generar propuestas pedagógicas para estructurar la enseñanza a partir de las necesidades y los diversos contextos infantiles. (Mercado Maldonado y Montaño Sánchez, 2015). La atención integral de la infancia se mira desde la diversidad y la relación de familia, docentes e instituciones responsables en la educación inicial

La transformación de las prácticas pedagógicas de las MC, es evidente en el detalle de los procesos, en la evaluación especialmente

las prácticas evaluadoras de las madres comunitarias están centradas en la vida del niño, en su interrelación social y su acción sobre el mundo. La ponderación se realiza con cada niño, y se basa en decisiones subjetivas, en los hogares comunitarios no existe la asignación de cifras y las ponderaciones se establecen mediante seguimientos colectivos en que se construye una actividad grupal (Zabala Archila, 2006, pág. 60).

Las MC “están generando un modelo pedagógico con un saber enriquecedor y liberador, dado por la socialización que teje un nuevo tipo de relación, transforma las realizaciones y las expectativas de estas educadoras" (Zabala Archila, 2006)

Es vital la sistematización de las experiencias vividas por las MC en su rol de educadoras y también en el rol de formación profesional y de producción de conocimiento (Macías Macías, 2015). Esta postura permite evidenciar la transformación de las prácticas educativas mediante procesos de construcción crítica de acciones, desde saberes y experiencias, en compatibilidad con el enfoque ecológico y sistémico de Bronfenbrenner (1979). 
Un tipo de formación que es necesaria y que han recibido algunas MC, lo constituye la formación en derecho para la solución de conflictos. En esta experiencia de formación las MC jugaron un papel silencioso, pero eficaz en lo concerniente a la solución básica de conflictos en las comunidades, hecho que no es fácil por estar ellas inmersas en un contexto conflictivo que altera la mediación y en el cual es difícil incidir debido a los intereses y motivaciones de poder que están en juego. Sin embargo, Builes (2013) resalta en las MC una postura no de choque y enfrentamiento con la problemáticas, sino de acomodación, no toman parte en la situación de forma directa, pero si inciden en la generación de espacios de convivencia.

La formación teórico conceptual y práctica que deben poseer las MC, para cumplir con la Política Pública de Primera Infancia, es el conocimiento de una cadena de servicios desde la gestación a los 5 años: servicios orientados a la mujer gestante, los servicios orientados a los niños durante el primer año de vida, los servicios para niños entre el primer año y el tercero; $y$ cuarto, la atención para niños entre los 3 y 5 años de edad. Este conocimiento debe seguir de cerca los lineamientos técnicos previstos para la atención a través de la estrategia De Cero a Siempre de la Comisión Intersectorial para la Atención a la Primera Infancia (2012)

Las necesidades de formación pedagógica y didáctica de las MC, están relacionadas con la labor que desempeñan y sus expectativas. Se comprometen fácilmente en la formación didáctica y pedagógica, dice Mongua Santafé (2008) y las experiencias se conciben como una oportunidad de superación y mejoramiento de la calidad

Asimismo, algunas investigaciones se han preguntado si los procesos formativos transforman las concepciones y prácticas de las MC sobre la atención a la primera infancia con discapacidades y/o alteraciones en el desarrollo, y se han interrogado sobre las necesidades de formación de las MC en los temas de discapacidad, alteraciones en el desarrollo y las estrategias para cualificar esta formación. Las respuestas esperan impactos sociales (Cuero Cordoba, Alvarez Sánchez, Granda Posso, y Jimenez Avilez, (2014))

También, la necesidad de plantear el avance en prácticas y saberes de las MC, se relaciona con la pregunta por el proceso de apropiación de los saberes compatibles con el desarrollo integral de la infancia; dado que, antes de plantearse la profesionalización, las madres fungían como "cuidadoras" categoría que Zibecchi (2013) define como "el trabajo de cuidar en tanto objeto de investigación, aún cuenta con ciertos vacíos de conocimiento" ( $p$. 2 ), y es necesario conocer el funcionamiento de las organizaciones sociales y comunitarias, su orige.n de mujeres proveedoras de cuidado, sus prácticas y saberes (Zibecchi, 2013). 
La formación de MC desde el Instituto Colombiano de Bienestar Familiar, contempla varias etapas desde el ingreso con requisitos y perfil de agente educativo, en una de las etapas se desarrolla un taller de capacitación inicial, que incluye temas básicos como: funcionamiento del servicio, responsabilidades, proceso de atención desde la perspectiva de derechos y deberes y componentes de modalidad como: Salud, alimentación y nutrición, desarrollo psicosocial y participación comunitaria, proceso pedagógico, formación a las familias y ambientes educativos protectores, entre otras responsabilidades de las MC para el cumplimiento de sus funciones en el marco de la formalización de mínimo 60 horas y pasantía, proceso de capacitación que los aspirantes realizan en un Hogar Infantil.

Es necesario conocer la comprensión y acciones que las MC tienen de los entornos, entendidos estos como espacios físicos y culturales donde habitan e interactúan los seres humanos y el contexto que les rodea. Son estos entornos los que harán posible el pleno desarrollo de los derechos por medio de condiciones humanas materiales y sociales, que deben ser explorados por las MC para promover la vinculación de diferentes actores responsables de la gestión y la atención integral, mediante su identificación y promoción por parte de los estudiantes en formación, estos entornos son: Entorno hogar, entorno salud, entorno educativo y entorno espacio público (ICBF, 2010)

Los procesos de cualificación direccionados al desarrollo del entorno educativo y su articulación con los demás entornos, se hacen por medio de la Ruta Integral de Atención, la cual debe ser ampliamente identificada y gestionada por las docentes y los demás agentes en este entorno. Otro aspecto importante a considerar es explorar, conocer y dominar los principios pedagógicos, en torno a la flexibilidad, para que sean abiertos en los métodos, contenidos y estrategias pertinentes para todos los actores, saberes, experiencias, contextos y prácticas involucradas, referidas a la contextualización particular de los participantes y sus necesidades.

Cualificar el talento humano en primera infancia es esencial para la calidad de la atención integral y gestión con esta población, en una perspectiva coherente a las políticas públicas y en concordancia en la implementación en la atención, programas y servicios para reconocer a los infantes como sujetos de derecho a través de espacios de diálogo desde sus diferentes formas de desarrollo infantil. Estos procesos de mejora requieren de una gestión intersectorial y articulada, con un enfoque diverso que favorezca el intercambio de saberes, experiencias y prácticas para enriquecer la construcción colectiva del conocimiento y la visualización de intenciones que den sentido, valor y razón a las prácticas de los agentes educativos. 
De esta forma, los propósitos que validan el conocimiento y las prácticas en la cualificación del talento humano que labora con la primera infancia se centran en: entender los niños y las niñas como sujetos de derecho, reconocer las interacciones entre adultos e infantes en la promoción del desarrollo infantil, abordar la diversidad de la infancia, las familias y comunidad en los proceso de atención integral, reconocer que las prácticas basadas en la gestión y la atención son herramienta esencial para abordar las dinámicas de la primera infancia, desarrollar las capacidades de los agentes educativos para propiciar ambientes de aprendizaje que favorezcan las competencias infantiles, conocer las políticas públicas y así beneficiar el proceso de desarrollo, la atención integral y asegurar la calidad de vida de los infantes, en torno al desarrollo de habilidades de gestión y cooperación para enfocar el trabajo desde la integralidad, a partir de la voz de los diversos actores. (Infancia, 2012)

Por lo anterior, la investigación: "Seguimiento a la formación de Madres Comunitarias a partir del programa de Pedagogía Infantil", se planteó como una experiencia investigativa para evaluar la pertinencia de la formación de las MC desde el programa de la Corporación Universitaria Iberoamericana CUI, en contraste con la formación misma a través de la fundamentación de los saberes específicos para la atención a la infancia delineada por la política pública y de las prácticas "in situ" con los niños y las niñas.

\section{METODOLOGÍA}

El alcance de la investigación fue descriptivo con enfoque mixto. El universo poblacional del estudio estuvo conformado por un grupo de MC de Hogares Comunitarios de Bienestar inscritas en el programa de formación Licenciatura en Pedagogía Infantil de la Institución de Educación Superior; uno de los instrumentos de investigación previstos en el estudio para la descripción de saberes y prácticas fue la entrevista a grupo focal para obtener la información. El instrumento utilizado fue diseñado a partir de los ejes nucleares que fundamentan los propósitos de la formación en los conocimientos teórico-prácticos para la atención y educación integral de la primera infancia en Colombia, y cumplen el objetivo de contrastar las prácticas y saberes de las MC con los fundamentos del Programa de formación en el cual están inscritas las docentes en formación.

Realizados los grupos focales, las transcripciones de las respuestas a las entrevistas y se analizaron a través de las categorías definidas según la formación y el alcance de la investigación para la detección de saberes y 
prácticas de las MC en sus centros de labor. Este análisis se sistematizó a través de matrices de resultados enmarcadas en las siguientes categorías, que se definen a continuación:

\section{TABLA 1}

\section{Definición de categorías según política pública de atención a la Primera Infancia en Colombia}

\begin{tabular}{|c|c|}
\hline Categorías & Definición para la investigación \\
\hline Concepción de Infancia & $\begin{array}{l}\text { Entendida en el marco del reconocimiento de los niños y las niñas } \\
\text { como sujetos de derechos y considerar la importancia de la interacción } \\
\text { con el adulto (madres comunitarias, agentes educativas, docentes } \\
\text { de primera infancia) para promover el desarrollo integral infantil. }\end{array}$ \\
\hline Prácticas Pedagógicas & $\begin{array}{l}\text { Circunscritas en la perspectiva de desarrollo infantil al diseño planea- } \\
\text { ción y gestión de proyectos de atención integral, sistematización de } \\
\text { los procesos, la práctica de una metodología a partir de las estrategias } \\
\text { y didácticas pedagógicas aplicadas para la gestión y el fomento del } \\
\text { desarrollo integral de la Primera Infancia. }\end{array}$ \\
\hline $\begin{array}{l}\text { Intersectorialdad e Inte- } \\
\text { gralidad }\end{array}$ & $\begin{array}{l}\text { En la atención y la formación, comprendida como el trabajo colabo- } \\
\text { rativo de las diferentes instancias e instituciones, referido a partir de } \\
\text { las políticas territoriales, nacionales e internacionales con relación } \\
\text { a los niños, niñas, familias y comunidades. }\end{array}$ \\
\hline Proyectos Innovadores & $\begin{array}{l}\text { Asumidos para este estudio como las experiencias, proyectos y/o } \\
\text { prácticas que generen nuevo conocimiento para los niños y las } \\
\text { niñas a través de propuestas pedagógicas significativas, creativas } \\
\text { e innovadoras. }\end{array}$ \\
\hline $\begin{array}{l}\text { Políticas Públicas en } \\
\text { Infancia }\end{array}$ & $\begin{array}{l}\text { Enmarcada en los lineamientos y políticas estatales a nivel local, } \\
\text { nacional e internacional sobre Primera Infancia, }\end{array}$ \\
\hline
\end{tabular}

Fuente: Documento No. 19 Referentes técnicos para la cualificación del talento humano que trabaja con la primera infancia.

La entrevista a grupos focales se realizó con base en un guion semiestructurado de preguntas relacionadas con los núcleos de formación de los referentes técnicos para la educación inicial en el marco de la educación integral para la infancia del ICBF y Mineducación.

Las preguntas de guía para las entrevistas a grupos focales giraron alrededor de la concepción de niñez que guía las prácticas de las docentes, los objetivos que orientan las prácticas pedagógicas desarrolladas, los lineamientos curriculares orientadores de esas prácticas pedagógicas, el proceso del trabajo pedagógico con los infantes, los conocimientos, prácticas y saberes sobre el desarrollo infantil, la información sobre la intersectorialidad y la integralidad necesaria para la atención a la niñez, la generación de alternativas y formas de atención innovadoras en el marco de las políticas públicas con enfoques poblacionales y territoriales. 
La composición del guion de entrevista se configuró, luego de la validación de contenido, por parte de pares evaluadores expertos con las siguientes preguntas:

1. La idea de infancia ha cambiado, antes se concebían los niños y las niñas como seres sin razonamiento, hoy se les considera seres con pensamientos, emociones y derechos. ¿Qué opinan sobre el tema de infancia comparando las ideas del pasado con las ideas de hoy? (Concepción de Infancia)

2. La práctica pedagógica se entiende como el conjunto de acciones educativas orientadas a la atención y el desarrollo integral de la primera infancia, así como las estrategias, los recursos, las intencionalidades, los objetivos y los contenidos que el docente programa, planea, desarrolla y evalúa.

¿Cuáles son los objetivos que orientas sus prácticas en las acciones pedagógicas con los niños y las niñas de primera infancia? (Práctica Pedagógica)

¿Cuáles son los lineamientos curriculares que orientan sus prácticas pedagógicas?

¿Hay algún proceso que deba seguirse en el trabajo pedagógico con los niños y las niñas de primera infancia? (Práctica Pedagógica)

Estas prácticas igual que las concepciones cambian con el tiempo, ¿Cuáles serían los componentes fundamentales que orientan sus prácticas para lograr el desarrollo integral de las niñas y los niños en la actualidad? Enuncien algunos procesos fundamentales que ustedes llevan a cabo, en el marco de la atención infantil.

3. El desarrollo de los niños requiere la atención a los aspectos físicos, psicológicos, sociales y familiares. (Intersectorialidad e Integralidad)

¿Qué instituciones atienden estos aspectos de los niños y las niñas con los que usted trabaja? y ¿Cómo se articulan estas instituciones para la atención?

4. El mundo moderno, el desarrollo del conocimiento y de las tecnologías propone la generación de formas alternativas innovadoras en todos los campos del desarrollo humano. (Práctica y/o proyectos innovadores). ¿Qué proyectosy/o prácticas innovadoras ha desarrollo con los niños y niñas con quienes ustedes trabajan? Mencione el título y el objetivo del proyecto y/o práctica. 
5. La Política Pública orienta unos ejes temáticos particulares que fortalecen la gestión y la atención integral de la primera infancia. Para explorar sus reflexiones, a continuación, se enuncian algunos ejes para que ustedes precisen su contenido: Atención Integral, Preservación de la vida de forma digna, Favorecimiento de los vínculos de los niños consigo mismos, su familia y sus cuidadores, Promoción de la autonomía e interacciones significativas, Características de los ambientes de aprendizaje, Clases de entornos para el desarrollo integral y características.

\section{RESULTADOS}

Los datos obtenidos a partir de las preguntas expuestas en el guion de entrevista a grupo focal 1 (en adelante GF1) y grupo focal 2 (en adelante GF2) aplicados a las MC, teniendo en cuenta las categorías contempladas para dar cuenta del objetivo planteado sobre determinar los saberes y las prácticas de las agentes educativas respecto a la atención y desarrollo integral de los niños y las niñas, son los siguientes:

\section{TABLA 2}

Síntesis Sistematización entrevistas a Grupo Focal Madres Comunitarias - Prácticas y Saberes -

\begin{tabular}{|c|c|}
\hline & Síntesis Interpretativa \\
\hline 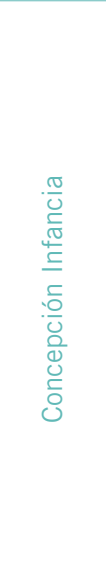 & $\begin{array}{l}\text { Concepción basada en los derechos y deberes, desde la libertad, la expresión y la } \\
\text { participación de los niños y las niñas. Educación desarrolla autonomía, valores, } \\
\text { espontaneidad y autoestima, además de las necesidades básicas de alimentación, } \\
\text { salud, vestuario y protección. Los discursos de las Madres Comunitarias están referi- } \\
\text { dos a estas características específicas que denominan la concepción de infancia. La } \\
\text { concepción de infancia, para las madres comunitarias ha cambiado totalmente, por } \\
\text { ejemplo el tipo de juegos que hoy practican los niños son más individuales, anterior- } \\
\text { mente los juegos eran más sociales, los tipos de juegos hacía que socializarán más, } \\
\text { eran más democráticos, no importaba la edad, la estura ni la clase social, todos se } \\
\text { divertían con "tin, tin, corre, corre" podrían jugar hasta niños o niñas que aparecían } \\
\text { en el momento del juego. Concepción del niño inocente, puro.... También hay una } \\
\text { concepción de niñez desde la "pérdida de la inocencia" confundida con la "ganancia } \\
\text { del conocimiento" Dicen las madres comunitarias en profesionalización. Otro aspecto } \\
\text { de la concepción de infancia es que constituye la etapa en que se debe construir con } \\
\text { libertad. A la pregunta por el objetivo de hacer estas actividades prácticas con los } \\
\text { niños, se afirman en querer que los niños participen, que descubran que pueden hacer } \\
\text { muchas actividades con libertad y que es muy importante participar en el mundo. }\end{array}$ \\
\hline
\end{tabular}




\begin{tabular}{|c|c|}
\hline $\begin{array}{l}\text { Cate- } \\
\text { gorías }\end{array}$ & Síntesis Interpretativa \\
\hline 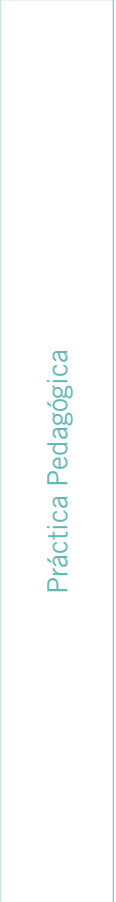 & $\begin{array}{l}\text { Enfoque Curricular: Proyecto Pedagógico Educativo Comunitario - PPEC- del ICBF, } \\
\text { Momentos pedagógicos: a) Bienvenida b) Vamos a Jugar, c) Vamos a Explorar, d) } \\
\text { Vamos a Crear. } \\
\text { Las estrategias pedagógicas: juego, teatro, literatura, la lúdica, talleres. } \\
\text { Contenidos: nomas de comportamiento, la naturaleza, los medios de comunicación } \\
\text { y de transportes; subtemas como los colores, deberes, derechos y otros considerados } \\
\text { necesarios, por medio de rincones, talleres y la ludoteca. } \\
\text { Recursos pedagógicos: películas, videos, cuentos, carteleras. } \\
\text { Evaluación: Escala cualitativa de valoración por edades y observación periódica con } \\
\text { dimensiones de valoración comunicativa, social, ética, corporal, cognitiva y afectiva } \\
\text { y criterios: estado en riesgo, en proceso o espera, y en estado avanzado. Se detectan } \\
\text { fortalezas y debilidades y se plantean mejoras. (Contempla los elementos del proceso } \\
\text { pedagógico, orientado, con seguimiento continuo y enfocado a favorecer el desarrollo } \\
\text { integral y la calidad de vida de la infancia.). } \\
\text { A la pregunta por el objetivo de hacer estas actividades prácticas con los niños, } \\
\text { se afirman en querer que los niños participen, que descubran que pueden hacer } \\
\text { muchas actividades con libertad y que es muy importante participar en el mundo. } \\
\text { A la pregunta por el objetivo de hacer estas actividades prácticas con los niños, se } \\
\text { afirman en querer que los niños participen, que descubran que pueden hacer muchas } \\
\text { actividades con libertad y que es muy importante participar en el mundo. Las prác- } \\
\text { ticas pedagógicas están basadas en lineamientos técnicos, pedagógicos y didácticos } \\
\text { orientados por organismos gubernamentales que consolidan la política pública, para } \\
\text { orientar el desarrollo sobre los pilares de la infancia: La importancia de la literatura, } \\
\text { la exploración del medio, el desarrollo del cuerpo y el juego. }\end{array}$ \\
\hline 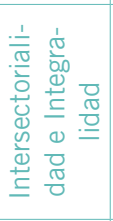 & $\begin{array}{l}\text { Existen apoyos a la infancia y las en los siguientes aspectos: sociales, psicológicos y } \\
\text { físicos desde las políticas nacionales y territoriales en primera infancia. ICBF, Bom- } \\
\text { beros, Comisarías de Familia, Secretaría de salud, Instituto Distrital de Recreación y } \\
\text { Deporte, IDRD, Emermédica (en algunas instituciones).(En términos generales falta } \\
\text { articulación e interacción intersectorial que garantice la integralidad y el restable- } \\
\text { cimiento de los derechos) }\end{array}$ \\
\hline 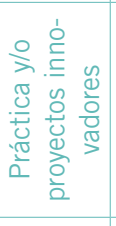 & $\begin{array}{l}\text { Proyectos: Huerta y reciclaje, objetivo hacer felices a los niños, promover su partici- } \\
\text { pación y la de sus familias, favorecer la espontaneidad, la imaginación, la seguridad } \\
\text { y la creatividad (los proyectos realizados reflejan una perspectiva según las necesi- } \\
\text { dades del contexto educativo, pero aún carecen de coherencia entre los discursos } \\
\text { y las prácticas dirigidas al mejoramiento de la didáctica, pedagogía e innovación, } \\
\text { direccionado al desarrollo integral de la infancia en el país. }\end{array}$ \\
\hline 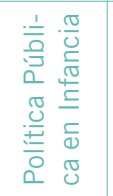 & $\begin{array}{l}\text { Política de Cero a Siempre, Pilares de la educación inicial: juego, didáctica, literatura y } \\
\text { exploración cognitiva. Ambiente de aprendizaje: espacio de adquisición conocimientos. }\end{array}$ \\
\hline
\end{tabular}

Fuente: elaboración propia

En términos de los saberes y en torno a la categoría concepción de infancia, las MC como agentes educativas de primera infancia señalan sobre la infancia, que en la actualidad el niño es un individuo que tiene la libertad de expresarse y participar en distintos escenarios de la cotidianidad, contrastado 
con épocas previas en las cuales este hecho no era permitido por el adulto ni la sociedad. Una MC, refiere (MC1-GF1): "los niños pueden manifestar lo que sienten, ser escuchados y participar en conversaciones de adultos".

Así mismo, las MC hablan de la importancia que los adultos han dado en esta contemporaneidad a la formación integral de la infancia y la educación completa desde la infancia hasta la universidad, ya que antes el adulto le daba más importancia a suplir las necesidades básicas del niño como el vestir, alimentar, la salud y cuidado. Así como lo manifiesta una (MC4-GF1): "anteriormente, los padres creían como atender a los niños en sus cosas básicas alimentación, vestuario y cuidarlo, ahí tenerlos en la casa... muy pocos eran los que iban a un jardín en ese tiempo, muy pocos fueron los que lograron sacar, ... pues pocos los que lograron hacer toda la primaria y el bachillerato".

La concepción de infancia se centra en el respeto a los derechos de la infancia, el reconocimiento del niño como un individuo autónomo, que requiere de cuidado, atención y formación en valores. Para estas agentes educativas (MC5-GF1): "Ios padres por medio de las madres comunitarias, las diversas instituciones y la tecnología se han enterado de los derechos que tienen los niños y han mejorado muchísimo, han formado a los niños como personas autónomas, personas con valores porque depende de esa formación y del cuidado que uno como sea, el papá, sea la mamá o nosotras como docentes que estamos en el proyecto de primera infancia, formamos a esos niños".

Los conocimientos sobre intersectorialidad e integralidad, entendida la primera noción como la necesidad que tienen los participantes en los procesos de cualificación de articularse con las políticas públicas a nivel local y nacional, contando con la colaboración de la familia, las comunidades y los distintos actores responsables del desarrollo integral de la primera infancia, se direcciona a promover el trabajo cooperativo en contextos de gestión de la política pública en torno a la garantía de los derechos de la infancia y la construcción de saberes y prácticas. En este sentido, las MC manifiestan que algunas entidades cuentan con el apoyo de psicólogos, el ICBF y otras entidades para abordar situaciones con la familia y favorecer procesos de desarrollo de los niños y las niñas. Así lo manifiesta una de las (MC2-GF1): "[...] a eso iba que parte interna esta la psicóloga, ella digamos así maneja trata diferentes temas hasta cierto punto, luego son remitidos a ICBF o sea depende del caso, si directamente que afecta al niño entonces se remite a ICBF en otros casos pues como se hacen visitas a las familias pues entonces ella ya detecta problemas ya en la familia entonces ella ya los orienta con la comisaria de familia". 
Así mismo, reconocen la integralidad desde los procesos contextuales de la infancia en torno a la diversidad y con el enfoque de pertinencia en torno a la garantía de los derechos y características particulares de los infantes. En general, no se precisa el concepto de integralidad de forma clara, ni incorporado a una práctica pedagógica diaria que garantice dicho desarrollo.

En cuanto a los conocimientos sobre las políticas públicas de infancia y la importancia en la articulación con las prácticas y saberes, siguiendo las disposiciones estatales para la atención y el desarrollo integral de la primera infancia, las agentes educativas manifiestan conocer la Estrategia de Cero a Siempre (2013) la cual enmarca la atención integral desde el aspecto nutricional, desarrollo infantil, provisión, salud, atención, recreación y pedagogía en el enfoque de derechos de los niños y las niñas. Una atención integral que entienden según relatan las (MC4-GF1): "integral seria, como en todos los aspectos del niño; aspecto nutricional". Estas apreciaciones referidas no solo a la atención de las necesidades básicas, sino de desarrollo del infante.

En cuanto a las prácticas pedagógicas las MC explican que desarrollan un planeador diario y semanal, centrado en momentos pedagógicos establecidos en al Proyecto Pedagógico Educativo Comunitario - PPEC - del Instituto Colombiano de Bienestar Familiar y los cuales son: a) Bienvenida, momento de motivación enmarcado en cantos y socialización de sentires y emociones de los niños y las niñas. b) Vamos a Explorar, momento previsto para el aprendizaje, la adquisición de conocimientos y relación de nuevos y previos saberes de los infantes, c) Vamos a Crear, momento para la creatividad, imaginación y planteamiento de estrategias y didácticas como los rincones, el cuento, la lectura, el teatro, el juego, la lúdica que permiten desarrollar la imaginación y el gusto por conocer mundos y realidades nuevas que fortalecen la autonomía, la libertad y la exploración. Así como lo menciona una MC (3M3-GF2), habla sobre el Proyecto que desarrollan: "el objetivo de las prácticas pedagógicas, para algunas madres comunitarias es realizar unas actividades con el propósito de conocer más los niños y también permitir el libre desarrollo para conocer el entorno de una manera divertida".

Teniendo en cuenta el PPEC del Bienestar Familiar las MC se reúnen para crear un Proyecto Educativo en cada Hogar Comunitario según lo mencionan las MC (1-M-1-GF2): "Nosotros como asociación nos reunimos a principio de año y sacamos un proyecto. Entonces qué hacemos? el proyecto durante el año va a ser este, sacamos una planeación, sacamos fechas importantes, he, por estándares de calidad, a nosotros nos piden planes específicos". 
Sobre las estrategias, recursos, contenidos y evaluación, señalan que todas las acciones pedagógicas se realizan a partir de las necesidades de los niños y las niñas y por esta razón, los temas están centrados en las normas de comportamiento que son relevantes en la primera infancia, crean rincones, espacios de exploración y vivencia, cuentan con ludotecas y talleres con padres. Los contenidos planteados se basan en conocer el barrio, el cuerpo, los medios de comunicación, la naturaleza, los medios de transporte, los colores, los deberes y los derechos de la infancia. Recursos como televisor, videos, películas, juguetes, cuentos, carteleras, entre otros, son los utilizados para trabajar con los niños y las niñas. A las MC les interesa trabajar normas y conductas, (MC4-GF1) es importante: "por ejemplo: si nosotras vemos que ellos no tienen buenas normas de comportamiento en el comedor... pues las trabajamos.... de comportamiento de aseo pues todo eso lo vamos incentivando en el diario del niño todos los días y pues.... eso tiene un proceso y para evaluarlo, pues... al transcurso del tiempo pues nos damos cuenta si se cumplió el objetivo o no".

Las MC indican que la evaluación se realiza a partir de una escala cualitativa de valoración para elaborar un boletín de registro de procesos y avances de los niños, teniendo en cuenta una planeación y 12 indicadores de logros determinados para la valoración. Éste instrumento lo provee el ICBF y está establecido de acuerdo con la edad de los infantes. Este proceso evaluativo es una observación y seguimiento periódico que se realiza cada mes o tres meses, según la autonomía de la normativa en cada municipio y está enmarcada en la dimensión social, corporal, autoestima, autonomía, conocimiento de objetos, entre otras, los indicadores de valoración establecidos son: estado en riesgo, en proceso o espera, y en estado avanzado.

Sobre el abordaje de Proyectos Innovadores, las MC refieren que estos están propuestos para fortalecer sus conocimientos y prácticas con el fin de mejorar el desarrollo infantil. Estas versiones indican que las propuestas innovadoras están enmarcadas en proyectos como la huerta, el reciclaje y el medio ambiente, con el fin de que los niños y las niñas sean felices, se diviertan y aprendan a través del juego, el arte y la exploración del medio. Una (MC5-GF1) dice: "[...] la propuesta innovadora que tenemos en el Centro de Desarrollo Integral - CDI - es la huerta, allí los niños se les enseña a cultivar a manejar las comidas que se pueden sembrar, para el cuidado del abono, el abono orgánico, tenemos una máquina que nos regalaron las estudiantes del Colegio San Bartolomé que fue creada por ellas para el manejo de los residuos, los residuos orgánicos, entonces los niños van a la huerta.... se les enseña la semilla.... hacer el huequito para la semillita, y todo el proceso...". 
En cuanto al conocimiento de las políticas públicas los resultados mencionan que conocen los Lineamientos Técnicos de Educación Inicial y que son muy importantes para su labor (2M2- GF2): "Sobre las políticas públicas, las madres comunitarias reconocen que los lineamientos técnicos son la biblia para su trabajo". Así mismo, otra MC refiere (M7.GF2): "la política pública de la primera infancia nos habla obviamente del desarrollo integral y la protección de los niños y niñas desde la gestación desde la gestación hasta los seis años todos somos corresponsables, madre comunitaria, entes territoriales estado todos somos responsables de garantizarlos derechos de los niños y de las niñas, en los aspectos como dicen integral ...en todo alimentación.. salud... en todo".

\section{ANÁLISIS Y CONCLUSIONES}

En cuanto a los saberes y prácticas de las MC se puede deducir que la concepción de infancia de las agentes educativas está referida a concebir los niños y las niñas como sujetos de derechos, participativos, autónomos y con deberes. Así mismo, se infiere que reconocen entornos como el hogar, la salud, la educación y de interacciones con los otros que hacen parte del contexto infantil y son esenciales en el desarrollo. En varias MC se hace evidente el considerar la construcción de conocimiento de forma colaborativa entre el adulto y el niño, sin embargo, se presentan vacíos en la fundamentación, argumentación e importancia del concepto de niñez en la atención integral de la primera infancia.

La apreciación empírica de reconocimiento de los niños y las niñas como individuos participativos, que poseen capacidades se hace evidente, sin embargo, no es clara la precisión de reconocer la infancia desde sus singularidades, considerar las historias sociales y colectivas para construir perspectivas pedagógicas en educación inicial, y entender que la niñez está definida y asumida en cada sociedad de forma diferente, dadas las características particulares que configuran una naturaleza diversa en los distintos contextos sociales. Se observa una argumentación basada en un lenguaje básico, según el discurso planteado y la carencia de términos precisos y de orden científico.

Las prácticas pedagógicas de las MC están centradas en la Guía para la Implementación del Proyecto Pedagógico Educativo Comunitario-PPEC-del Instituto Colombiano de Bienestar Familiar - ICBF - (2011), documento que plantea los momentos pedagógicos como estructura de organización pedagógica (planeación, desarrollo, evaluación) teniendo en cuenta el tiempo y el espacio para desarrollar actividades formativas en cada intervención. Estos espacios 
son: a) Bienvenida, momento en el que se identifican el estado de ánimo de los niños y las niñas, cuando llegan al jardín, relatar experiencias previas, la disposición de acciones y actividades con el fin de socializar los temas definidos a trabajar cada día y de esta forma suscitar nuevos aprendizajes; b) Etapa de Exploración, espacio de reconocimiento, exploración, comprensión y desarrollo de capacidades de relación, que permiten la construcción del conocimiento y convoca a los infantes a identificar nuevos entornos, fenómenos, objetos, medios sociales, comunitarios y culturales de la cotidianidad que permiten fortalecer la observación y la deducción como habilidades básicas del aprendizaje; c) Espacio de creación, se basa en desarrollar la comunicación a través de los lenguajes expresivos y fomentar la capacidad creadora. En este momento es esencial la motivación de propuestas innovadoras por parte del agente educativo, con el fin de permitir en los niños y las niñas la expresión de sus ideas, intereses, necesidades, reconociendo sus conocimientos y saberes. Así mismo, estimular el desarrollo de las habilidades artísticas, el encuentro con el arte y la manifestación de sus diferentes expresiones. De igual forma, incentivar la apreciación estética a través de la sensibilidad y el reconocer las diversas expresiones simbólicas de la realidad; d) Espacio para juego, pretende animar al desarrollo de la imaginación, la creación de normas, estimular la comunicación y favorecer una vida en común armónica; e) Espacio de despedida y regreso al hogar, espacio de reflexión y concreción de aprendizajes, actividad de cierre enmarcada en la expresión de gustos y vivencias por parte de los niños y las niñas, la invitación a compartir los saberes del día con la familia desde una perspectiva lúdica.

Los momentos son mencionados por las MC en términos de la denominación propia de los espacios, sin embargo, no se evidencia una apropiación clara del enfoque pedagógico determinado en cada ambiente, en cuanto a la relación de la planeación, desarrollo y evaluación centrados en la atención integral de la infancia. De igual forma, se determina la aplicación de estrategias pedagógicas como el juego, el teatro, la literatura, la lúdica y los rincones temáticos, referidos como medios para acceder al conocimiento sin una contextualización de la importancia pedagógica y la metodología aplicada para el desarrollo integral de la infancia. El énfasis en los contenidos abordados está basado en las normas comportamentales, y temáticas de contenido como la naturaleza, los medios de comunicación y de transportes, evidenciándose la necesidad de realzar los procesos axiológicos. Los recursos pedagógicos utilizados son películas, videos, cuentos, carteleras, los cuales señalan la aplicación de elementos distintos a los tradicionales y con metodologías diferentes, así como utilizar recursos innovadores.

Los lineamientos del estado orientan la formación integral, para desarrollar las dimensiones por medio de procesos lúdicos. El objetivo de realizar diferentes actividades prácticas con los niños, es su participación, 
que descubran su capacidad y destrezas con libertad y autonomía. Luego, las prácticas pedagógicas están basadas en lineamientos técnicos, pedagógicos y didácticos orientados por organismos gubernamentales que consolidan la política pública, para orientar el desarrollo sobre los pilares de la infancia: La importancia de la literatura, la exploración del medio, el desarrollo del cuerpo, denominaciones señaladas por las MC, sin una precisión de nombres.

Aunque la evaluación se hace a partir de una escala cualitativa de valoración por edades y observación periódica del desarrollo, con criterios valorativos: Estado de riesgo, en proceso o espera y en estado avanzado, aún no se evidencia de forma clara una sustentación de evaluación argumentada desde el enfoque pedagógico y de desarrollo del infante desde la multidimensionalidad, aún no se evidencia un proceso minucioso de observación del desarrollo infantil y el de las dimensiones del ser humano, mediado por el campo educativo desde la relación del niño con los entornos de socialización y sus interacciones cotidianas.

Las MC entienden ambiente de aprendizaje como un espacio de conocimiento básico, sin especificar factores o aspectos relevantes y pertinentes. Son pocas las experiencias que señalan el aspecto de la diversidad como un elemento esencial a considerar en la atención y desarrollo integral de la infancia, no se refieren experiencias pedagógicas significativas en este aspecto. De igual forma, no se evidencia una observación y seguimiento en el marco de la valoración que haga parte de una planeación o metodología mediada por didácticas y estrategias.

La Intersectorialidad e integralidad está abordada desde las orientaciones dadas por la política pública, sin articulación específica en la práctica y de forma generalizada y se cuenta con la articulación de entidades como el Museo de las Ciencias y el Juego, el Centro Zonal y la Alcaldía Municipal, la Secretaría de Salud, los Bomberos y el apoyo de las psicólogas para el apoyo de las acciones encaminadas a la atención y desarrollo integral de la primera infancia. Este panorama en el aspecto intersectorialidad e integralidad muestra un desarrollo incipiente, a partir de los datos suministrados por las MC y dado que algunas entidades de atención infantil cuentan con estas alianzas o vínculos para dar cumplimiento a la disposición de asegurar la salud, la educación, la recreación y los derechos de la infancia a través de convenios, gestiones y organización institucional como actores responsables de la infancia.

En este sentido, se hace visible el aporte de los organismos sectoriales en términos de la atención en salud suministrada por los hospitales, con servicio médico odontológico, psicología, así como visitas domiciliarias a las familias. Se realizan evaluaciones periódicas a los niños, por ejemplo 
evaluación de peso y talla. Asimismo, otras entidades como Bomberos y la Cruz Roja suministran charlas y conferencias para mejorar los conocimientos sobre la salud y los derechos básicos de los niños y niñas. No obstante, los organismos sectoriales realizan una labor con la infancia, esta se realiza con eficacia con la dinamización de parte de las MC, quienes realizan conjuntamente planes y programas de atención a la niñez. Al parecer, faltan los recursos para gestionar salidas pedagógicas y formación cultural y social. Asimismo, es necesaria la formación para la gestión de los agentes educativos para dirigir la articulación efectiva de los organismos del estado en salud, recreación, derecho y de familia, entre otros, para garantizar el desarrollo integral y una atención multidimensional a los infantes.

Los proyectos pedagógicos, que realizan las MC son de carácter contextual y cotidiano, con necesidad de profundización del saber sobre los lineamientos nacionales e internacionales para atender de forma integral a la primera infancia, como se recalca en el punto anterior. En general, se visualiza una proyección pedagógica innovadora incipiente, dado que, se presenta un avance discreto en la innovación de proyectos desarrollados con la finalidad del desarrollo y mejora de la calidad de vida de los niños y las niñas y la garantía de formación para el restablecimiento y respeto de derechos de la infancia. Se infiere que la innovación se asume como nuevas prácticas y proyectos que no se habían realizado antes y que al realizarlos ahora se convierten en una nueva propuesta en el contexto del aula.

Proyectos pedagógicos de aula como la huerta, la casa mágica, el medio ambiente, los colores, los animales y la familia, entre otros, desarrollados a través de la danza, la música, el arte, el juego, la exploración del medio hacen parte de las experiencias pedagógicas significativas que realizan con los niños y niñas, las agentes educativas. Si bien se desarrollan nuevas prácticas pedagógicas contextualizadas con las necesidades y realidades de la infancia, se deduce una ausencia de articulación con las metas y componentes pedagógicos referidos a didácticas y estrategias innovadoras que transformen los ambientes de aprendizaje y permitan el acceso al conocimiento y favorezcan la atención integral de la infancia.

Los proyectos que se están propiciando desde los ministerios son por ejemplo los proyectos tecnológicos con las docentes, pero no se han podido llevar a cabo con los más pequeños, ya que los proyectos que se impulsan están de acuerdo con los recursos que hay en cada Hogar Infantil, por ejemplo computadores, hasta ahora se han llevado a cabo proyectos del uso de medios como la televisión. Hay proyectos sencillos y tradicionales, concebidos como innovadores según las temáticas que se trabajan con los niños y los contextos, pero es necesario fortalecer este aspecto para dar impulso a la educación que continúa en la educación primaria. 
El conocimiento de las Políticas Públicas en Infancia, que poseen las $\mathrm{MC}$ en profesionalización, se percibe como un saber nocional adquirido y dominado en sus principales objetivos y metas, como se describe en la Estrategia de Cero a Siempre (2013), en el marco de la Política Pública de Primera Infancia (2007) y el Lineamiento Pedagógico y Curricular para la Educación Inicial en el Distrito. Bogotá. D.C (2013), documento en el cual se refieren los Pilares de la Educación Inicial, lineamientos divulgados ampliamente por lo entes educativos y gubernamentales. Es evidente la falta de precisión en los nombres específicos de los lineamientos políticas estatales sobre infancia, aunque se mencionan postulados teóricos de las experiencias sobre las dimensiones de desarrollo del ser humano y la apropiación de la cultura, A partir de este panorama, se puede entender que el desarrollo integral de los niños y las niñas es una de las políticas públicas que se debe tener en cuenta en la formación de agentes educativos para la infancia. También la preservación de la vida, con todo lo que esto conlleva y restablecimiento de derechos a nivel de alimentación, nutrición, cuidado, recreación y salud. En términos generales las MC responden que saben lo que deben hacer, pero que no saben definir los avances en torno a la infancia, y que por esta razón están estudiando para saber más sobre este aspecto.

De este análisis surge la necesidad de fomentar indagaciones similares y continuar la evaluación de la formación de agentes educativas de la infancia, específicamente agentes educativos, cuyo formación específica profesional está orientada a la formación y calidad de vida infantil.

\section{BIBLIOGRAFÍA}

Blanco Vega, M. d. y Arias Castilla, C. A. (2015). Seguimiento a la formación de madres comunitarias a partir del Programa Pedagogía Infantil a Distancia de la CUI. Corporación Universitaria Iberoamericana, Cundinamarca. Bogotá, D.C.: Facultad de Educación, Ciencias humanas y sociales de la Corporación Universitaria Iberoamericana.

Borjas, M. y Jaramillo, L. (2009). Transformaciomes alrededor del discurso prácticas educativas, actitudes y relaciones sociales de las Mades Comunitarias del ICBF. (U. d. Norte, Ed.) Psicología del Caribe(23), 113-132.

Bronfenbrenner, U. (1979). La ecología del desarrollo humano. Cognición y Desarrollo humano. Experimentos en entornos naturales y diseñados (Paidós ed.). (M. Cubí, Ed., \& A. Devoto, Trad.) Barcelona, Barcelona, España: Paidós.

Builes Correa, M. V. (5 de Diciembre de 2013). Reseña de Las redes del cuidado: madres comunitarias y vínculos que protegen. (U. d. Antioquia, Ed.) Revista de Psicología, 5(2), 127-129.

Cuero Cordoba, A. A., Alvarez Sánchez, G. E., Granda Posso, L. Y. y Jimenez Avilez, L. P. (2014). Acompañamiento formativo a madres comunitarias para la atención a niños y niñas con discapacidad y/o alteraciones en el desarrollo. UdeA, Antioquia. Medellín: Universidad de Antioquia. 
Guzmán, R. J. y Guevara, M. (2010). Concepciones de infancia, alfabetización inicial y aprendizaje de los educadores y educadoras. (C. d. juventud, Ed.) Revista Latinoamericana de Ciencias Sociales, Niñez y Juventud, 8(2), 861-872.

Hernández Cervantes, L. y Blanch, J. P. (2014). La enseñanza de las ciencias sociales en la educación infantil en México. Revista mexicana de investigación educativa, 30(1), 65-94.

Hernández Muñoz, L. M. (2013). Apropiación de las madres comunitarias en prácticas culturales significativas para la educación de los niños en Hogares de ICBF. Universidad Católica de Pereira-Especialización en Pedagogía Y Desarrollo Humano, Risaralda. Pereira: Universidad Católica de Pereira.

ICBF. (2010). Lineamiento Técnico Administrativo y Operativo modalidades hogares comunitarios de Bienestar. Instituto Colombiano de Bienestar Infantil, Cundinamarca. Bogotá, D.C.: ICBF.

Infancia, C. i. (2012). Cartilla Atención Integral : Prosperidad para la primera infancia. Alianza Mineducación, I.C.B. F. y Presidencia de la República, Cundinamarca. Bogotá, D.C.: Mineducación, I.C.B.F. y Presidencia de la República.

Infancia., C. i. (2012). Cartilla: Atención Integral. Prosperidad para la Primera Infancia. MineducaciónICBF-Presidencia de la República, Comisión intersectorial. Bogotá, D.C.: MIneducación.

Jaramillo, L. (2009). La política de primera infancia y las madres comunitarias. (I. d. Norte, Ed.) Zona próxima. Revista del Instituto de estudios en Educación de la Universidad del Norte, 2(11), 86-101.

López Lara, J. A. (2014). Madres comunitarias, relatos de vida y configuración de identidades en el marco del programa de hogares comunitarios de Bienestar. Pontificia Universidad Javeriana-Maestría Ciencias Políticas y Relaciones Internacionales, Cundinamarca. Bogotá, D.C.: Repositorio Pontificia Universidad Javeriana.

Macías Macías, C. Y. (2015). Practicas educativas de madres comunitarias: sistematización de experiencias. Pontificia Universidad Javeriana, Facultad de Humanidades y Ciencias Sociales. Cali: Pontificia Universidad Javeriana. Repositorio Vitela.

Martinez Garrido, C. y Murillo, f. J. (2016). Invetigación Iberoamericana sobre enseñanza eficaz. (R. M. educativa, Ed.) Revista Mexicana de investigación educativa, 21(69), 471-499.

Mercado Maldonado, R. y Montaño Sánchez, L. (2015). Procesos de participación entre profesoras de jardines de niños y madres de familia en actividades de enseñanza. (R. m. investigación, Ed.) Revista mxicana de investigación educativa, 20(65), 347-368.

Mongua Santafé, A. P. (2008). Necesidades pedagógicas y didácticas de las madres comunitarias del Barrio Benavista. Universidad de San Buenaventura, Bogotá, D.C. Bogotá: Universidad de San Buenaventura.

Osorio Franco, Y. A., Cortés Urrego, L. A. y Rodas Mejía, M. N. (2016). De madres comunitarias a agentes educativos: Experiencia y formación, vicisitudes de un trayecto. Universidad de Antioquia, Antioquia. Medellín: Facultad de Educación Universidad de Antioquia.

Soto Panesso, F. E. y Casanova Martínez, H. (2009). Madres comunitarias el ser y el hacer una construcción social. Universidad Tecnológica de Pereira, Risaralda. Pereira: Repositorio Universidad Tecnológica de Pereira.

Zabala Archila, J. C. (2006). Las madres comunitarias en Colombia. Investigación sobre la evaluación participativa. Universidad de Granada, Departamento de Didáctica y Organizacion Escolar. Granada: Universidad de Granada.

Zibecchi, C. (5 de Enero de 2013). Organizaciones comunitarias y cuidado en la priera infancia, Un análisis entorno a las trayectorias, práctuicas y saberes de las cuidadoras. (R. C. argentinas, Ed.) Revista Trabajo y Sociedad.(20), 21. 